\title{
Dust investigations at ASDEX Upgrade
}

V. Rohde, M. Balden, T. Lunt, and the ASDEX Upgrade-Team

Max-Planck-Institut für Plasmaphysik, EURATOM Association, Boltzmannstr.2, D-85748

Garching, Germany

PACS numbers: 28.52.Fa, 52.25.Vy, 52.70.Nc, 52.50.Hf,52.55.Fa

\section{Abstract}

Investigations on dust in full tungsten divertor tokamak ASDEX Upgrade are summarized. Newly designed collectors are used to distinguish plasma-produced dust from other kind of debris. Whereas sub-micron particles are often tungsten spheres, larger ones are agglomerates from tungsten in a boron/carbon matrix. Extrapolation of the measurement yields to $80 \mathrm{mg}$ of carbon and $1200 \mathrm{mg}$ of tungsten in the dust, including $510 \mathrm{mg}$ of tungsten spheres. In case of its mobilization, the amount of tungsten observed may constitute a significant impurity source. Droplets produced by arcs seems to be the production mechanism for the tungsten spheres. The strong light emission of dust particles penetrating the SOL are rarely observed by a fast camera during normal discharges, but very frequently after disruptions. As the particles follow straight lines, we infer only a weak interaction with the SOL plasma. This is also confirmed by the observation of fragile agglomerates. 


\section{Introduction}

The licensing procedure of ITER promotes the topic of dust in fusion devices [1], although in present experiments dust plays a minor role [2]. In this paper we will summarize the ongoing activities on dust at ASDEX Upgrade (AUG) and address in particular the tungsten first wall [3].

The most common way to collect dust is the so-called filtered vacuum technique [4]. In 2000, AUG used carbon plasma-facing components (PFCs) at this time, dust investigations applying these methods were started [4], $980 \mathrm{mg}$ of dust were collected in total. The major part $(94 \%)$ was found at the bottom of the vessel. Average count-based particle size from all locations was $3.3 \mu \mathrm{m}$. Detailed investigations of the collected dust were carried out by optical microscopy, scanning electron microscopy (SEM) and energy-dispersive X-ray spectroscopy (EDX) yielding a zoo of different shapes and chemical elements.

Their composition and morphology indicate that many particles were produced by in-vessel work and workers as well as by non-perfect components of in-vessel diagnostics. These sources are significant in AUG, even if the vessel is carefully vacuum-cleaned before each start of the experimental campaign. AUG has been in operating since nearly 20 years. Some redesigns of the in-vessel components had been performed, which required works like drilling and welding inside the vessel. One example of non-perfect design of diagnostics is the cabling of Langmuir probes, which uses ceramic fibres for isolation. If the probe current is swept during magnetic field operation, the cable moves and the isolating fibres crack releasing a large number of fragments.

In the following it is distinguished between dust (plasma-produced or plasma contact) and 
debris (everything else). As long as the debris is not mobilized and does not reach the plasma, it will have negligible influence on the plasma operation and the machine safety. Due to more careful and remotely handled in-vessel work as well as design of in-vessel components to mitigate dust, it is expected that debris will hopefully play only a minor role in ITER.

\section{Dust collection}

Since AUG operates with full tungsten PFCs, no thick deposited layers were observed anymore [5]. Thin layers were formed at the inner divertor, consisting of boron and carbon, but they are much too thin to produce flakes, as observed in the investigations with the carbon PFCs. To separate dust from debris, dust collectors were installed in the main chamber of AUG [6]. These consist of pieces of Si wafer for collection and a collar, which prevents dust to be removed from the wafer during vent. The collectors were mounted during a vent and protected by a cap, which was removed just before the closing the vessel and mounted again just after the venting. Up to 5 collectors at 4 different toroidal and 3 poloidal positions had been used since 2006 (see insert at Fig. 1). After removal, the particles collected on the Si were accurately evaluated by SEM and EDX.

In contrast to the filtered vacuum technique the collectors have to be mounted in advance and the dust is collected only at some discrete positions. However, this technique allows distinguishing clearly between dust and debris and is able to detect even tiny particles due to the highly sensitive analysis and the usage of mono-crystalline collectors. The advantages of the collectors are the separation of plasma-produced or mobilized dust from most of the debris, the conservation of the surrounding of the particles, the high probability to collect all particles, even very tiny ones, and the sensitivity for analysis due to the selection of mono-crystalline 
collectors.

\section{Classification of dust particles}

On the collectors some particles with a size of $100 \mu m$ consisting of carbon are found, which show deposited layers close by. From the geometry it is obvious that the deposition originates from these particles. The reason for the evaporation seems to be strong heating, i.e. overheated particles have been fallen onto the collector or particles have been heated until they start to evaporate. To estimate the role of this effect as carbon source in AUG, $50 \%$ erosion of the observed particles is assumed. The low number of observed overheated particles leads to a high statistical error. But as only some $m g$ of sublimated carbon is assessed this effect is negligible on the average in particular for the full tungsten AUG.

High resolution pictures with a pixel diameter of $0.125 \mu m$ of regions with a size of $442 \times$ $512 \mu m^{2}$ on the collectors are taken by SEM. The average size distribution of the particle is shown in Fig. 1. Due to finite resolution of the pictures used, this evaluation is restricted to particles larger than $0.25 \mu \mathrm{m}$. The size distribution shows a maximum for the smallest particle diameter and logarithmic decay, which becomes even steeper for particles above $5 \mu \mathrm{m}$. To confirm the huge amount of sub-micron particles, high resolution SEM pictures are taken. In Fig. 1 data from three collectors, exposed at the same poloidal and different toroidal positions, are shown. The fact that almost the same distribution is found in the 3 sectors confirms the assumption of toroidal homogeneity of the dust sources. Earlier investigations (carbon PFCs) at the same position yield a Gaussian distribution with a centre at $5 \mu \mathrm{m}$ and a half-width of $3 \mu \mathrm{m}$ [4]. Therefore, the huge amount of sub-micron particles seems to be related to the tungsten PFCs. 
To classify the particles, some regions on the collectors were arbitrarily selected and all particles within this area were magnified and analyzed using EDX. Small particles mostly consists out of one chemical element, whereas bigger one are often agglomerates. These data are used to derive the effective occurrence of the elements as compiled in table 1.

A significant difference in the morphology and contribution of particles with a diameter below and above $5 \mu m$ was observed. The results of these investigations are compiled in table 1. Most of the tungsten particles with a diameter below $5 \mu m$ are small spheres. Larger particles often show a more complex structure. Particles with a size larger than $50 \mu m$ are quite rare even if they dominate the total weight. The statistics is to poor to derive reliable elementary analysis.

\begin{tabular}{|c|c|c|c|c|c|}
\hline size & boron & carbon & iron & tungsten & other \\
\hline$<5 \mu m$ & $26 \%$ & $16 \%$ & $4 \%$ & $55 \%$ & \\
$5-10 \mu m$ & $21 \%$ & $30 \%$ & $12 \%$ & $21 \%$ & $15 \%$ \\
\hline
\end{tabular}

Table 1: Composition of dust particles collected during the campaign 2008.

The total amount of particles was estimated extrapolating of the collection area to the area of the horizontal plate at the low field side. If one normalizes the number of particles to the plasma discharge time, a particle production rate of $5 \cdot 10^{6} s^{-1}$ is found for all particles and of $5 \cdot 10^{4} s^{-1}$ for the larger ones $(>5 \mu m$ ). It has to be distinguished between tungsten and carbon, which behave quite different. The distribution showed in table 1 is used to get the total amount of material deposited as dust. Results for the second part of the 2008 campaign (\#23673-\#24189) are compiled in table 2. 


\begin{tabular}{|l|c|c|c|}
\hline & total tungsten & tungsten $<5 \mu \mathrm{m}$ & carbon \\
\hline amount of dust & $1210 \mathrm{mg}$ & $510 \mathrm{mg}$ & $80 \mathrm{mg}$ \\
impurity influx & $2.1 \cdot 10^{18} \mathrm{at} / \mathrm{s}$ & $9.0 \cdot 10^{17} \mathrm{at} / \mathrm{s}$ & $1.4 \cdot 10^{17} \mathrm{at} / \mathrm{s}$ \\
\hline
\end{tabular}

Table 2: Amount of impurities deduced from extrapolation of the dust quantities measured by the collectors.

The amount of carbon can be neglected with respect to the carbon turnover in AUG, which is about $1 \mathrm{~g}$ per campaign. Tungsten erosion at the divertor was measured by marker stripes [7]. Net erosion was found only at the outer divertor, where physical sputtering dominates. The amount of tungsten derived from dust data presents about $17 \%$ of the erosion at the outer divertor. Other tungsten sources as molten layers of coatings have to be taken into account, i.e. an even higher release of tungsten is expected. As most of dust particles contain pure tungsten, it is unlikely that tungsten found in dust particles had been eroded by physical sputtering. The amount of tungsten observed as dust particles can play a significant role as impurity source in AUG. Especially bigger particles have a high penetration probability at the SOL.

\section{Dust production by arcs}

The morphology of the collected particles has to agree with the dust production mechanism. Here we restrict ourselves on arcs, which were discussed in the 1980th as important impurity production mechanism in all metal devices [8]. The material release by arcs depends on the heat conduction and melting or sublimation point of the material [9]. As these properties are quite similar for tungsten and carbon, the eroded mass per charge agrees within a factor of two for both materials. 
Arc tracks had been also observed in AUG for carbon PFCs, but the amount of eroded material by arcs is negligible compared to the amount of carbon released by physical and chemical erosion. This is different in the case of tungsten due to the strongly reduced physical erosion. Arc erosion in AUG is not poloidally uniform: the arcs tracks observed concentrate in some regions, as the inner divertor baffle, the upper divertor baffle and the roof baffle [10]. At the inner divertor baffle up to $12 \%$ of the surface area of a tile is affected by arc traces [11]. The traces are toroidally distributed uniformly. On a single tile they are mostly located at deposition dominated regions, where the angle of impact of the magnetic field lines is very shallow and they are aligned perpendicular to the magnetic field lines. Arcs are known to produce droplets, which are obvious candidates for the sub-micron spheres observed on the collectors.

As the tungsten spheres show no contamination by other impurities, melting of tungsten layers seems to be the production mechanism. Unfortunately, some local macroscopic damage of the layers at distinct toroidal positions occurred in AUG during the exposure of the dust collectors. Molten layers typically produce droplets up to $\mathrm{mm}$ size or evaporate. Droplets produced by arcs are smaller than the arc trace depth: sub-micron droplets are expected for $3 \mu m$ thick tungsten layers as used in AUG. This expectation is confirmed by SEM investigations near the arc traces, which show many sub-micron tungsten spheres and re-solidified edges of the arc traces. Also, the arc traces and deposition of tungsten spheres are toroidally uniform. Therefore, arcs seem to be a plausible production mechanism of the sub-micron tungsten spheres observed.

Taking only the arc traces at the inner divertor baffle into account, a tungsten erosion of $2 \mathrm{~g}$ is expected. Assuming a conversion factor of 0.1 for the droplet production, $6 \cdot 10^{20}$ tungsten 
atoms in form of droplets are expected, whereas $1.7 \cdot 10^{21}$ tungsten atoms $(510 \mathrm{mg})$ were found as droplets ( diameter $\leq 5 \mu m$ ) in the collectors. The two values agree within the errors expected from the very rough evaluation method. The homogeneous orientation of the arc traces shows that the arcs are active during the presence of the magnetic field, i.e. not during the phases of glow discharge cleaning. If the droplets are produced and transported during plasma operation, only a small amount of them moves to the low field side (at the position of the collectors), without being eroded by the plasma. On the other hand, another region of arcs as the upper passive stabilization loop protection limiters (shown in the insert of Fig. 1) will produce additional droplets. In summary, the determined number and size of tungsten spheres fits to the picture of droplet production by arcs.

Larger dust particles show often a complex structure as shown in Fig. 2. Some particles are cut using a focused ion beam to get access to the internal structure. From EDX analysis the particles are identified as an agglomerate of a boron/carbon matrix filled with tungsten particles. To identify their origin, deposited layers from different regions on the PFCs are analyzed. Layers of almost the same morphology are found at the inner divertor baffle. These are located at positions close to the arc traces. The tungsten particles are presumably droplets from the arcs, which are embedded in the layers. Again, these layers are on the high field side, whereas the agglomerates are collected on the low field side of the plasma. If the particles were transported during a plasma discharge, they would enter the SOL plasma and the delicate structure as shown in Fig. 2 would have been damaged by erosion due to the plasma and so it could be speculated that also transport occurs when the plasma is not dense or even absent. 


\section{Time resolved measurements}

Collected dust provides only limit information on its production, transport and its behaviour in the plasma. Video cameras are available as standard diagnostics. Their time resolution is not sufficient to follow dust trajectories of fast anf small particles. But they detect dust events and so they can be used at least to identify regions, where dust is produced. This technique was developed for the DITS (deuterium in ToreSupra) campaign in ToreSupra, where dust events become more frequent with time of operation [12]. Applying the same software to AUG data reveals a complete different behavior. It is observed that most dust events (i.e. dust particles penetrating the SOL where they start to radiate strongly) occur during start-up of a campaign. The number of events drops during operation clearly indicating conditioning.

To follow dust trajectories and to measure the speed of the dust particles, a fast CMOS camera is used. The camera operates routinely at a frame rate of $10000 \mathrm{~Hz}$ viewing in the midplane tangentially into the vessel. After the initial conditioning only few dust events are observed. On the contrary, after disruptions many dust events are observed.

To visualize the trajectories of the particles in one picture, a sequence of 500 frames is selected and the maximal intensity for each pixel is plotted. Fig. 3a shows the resulting trajectories $0.1 \mathrm{~s}$ after a disruption. The exposure was only half of the time between two frames, which results in a stroboscopic effect (i.e. the dashed lines in the figure) on the trajectories. As most of the trajectories are straight lines the interaction of the particles with the remaining plasma is assumed to be negligible and the charge of the particles seems to be too low to cause a significant Lorentz force in the magnetic field. Low plasma interaction will not cause erosion, even on complex particles as the agglomerates (Fig. 2). To derive particle velocities information of the distance from the particle to the lenses is needed. To map the two-dimensional 
information obtained by the camera, a plausible distance is assumed. This evaluation yields particle velocities in the range of $10-100 \mathrm{~m} / \mathrm{s}$.

Another example for unusual dust events is shown in Fig. 3b. The data are taken during a discharge with a high clearance of the plasma from the limiters. Now strong interaction of dust particles with the plasma, acceleration, and splitting of particles is observed.

Dust particles are only visible by the fast camera, if they radiate more photons than the surrounding plasma. For typical SOL conditions a minimal diameter of $5 \mu \mathrm{m}$ and a temperatures above $1500 \mathrm{~K}$ are needed [13]. Such strong heating by the ambient plasma will cause significant erosion on the particles, which will destroy the delicate structure of the agglomerates (Fig.2). Extrapolating the results of the collector probes about $1 \cdot 10^{3} s^{-1}$ dust particles larger than $5 \mu \mathrm{m}$ are expected to enter the field of view of the camera each discharge. The number of dust events observed by the camera is orders of magnitude less, indicating that most of the particles are not heated high enough to be detected by the camera. This finding is in line with the morphology of the agglomerates collected. Camera observations yields that disruptions play a significant role at least in the release/transport of the dust particles. On the other hand the high clearance discharges indicate that more dust particles are available but not visible due to the high dense SOL for normal AUG discharges. The mechanism of release and transport of the agglomerates are still under investigation.

\section{Summary}

Dust was collected, quantified and characterized in the full tungsten wall tokamak ASDEX Upgrade. Special collectors were used to gather only particles produced or mobilized by the 
plasma. Whereas sub-micron particles are predominantly tungsten spheres, bigger particles often consist of an agglomerate of boron, carbon and tungsten. While the amount of $\mathrm{C}$ found in the dust particles is negligible, the tungsten particles may constitute a significant source of impurities. The tungsten spheres are presumably droplets produced by arcs on the tungsten coatings. The agglomerates show the same morphology as the deposited layers at the inner divertor baffle region.

Dust events are observed by a fast camera. Apart from the initial conditioning phase, dust is only observed after disruptions and in high clearance discharges. Since the trajectories after disruptions are straight lines, it could be infered, that the interaction of the particles with the plasma is only weak. The huge amount of agglomerates and their delicate structure point to the fact that most of them are not detected by the fast/standard video cameras. Fast camera observations shows dust events only after disruptions or high clearance discharges. The typical speed of the particles is $10-100 \mathrm{~m} / \mathrm{s}$.

Further dust collections are required to verify these results and additional effort is needed to understand the dust production and its transport. High clearance discharges will be investigated in the future to study the dust production in discharges without disruptions. The most important point seems to be the identification of the origin and the behaviour of the arcs.

\section{References}

[1] Roth J et al. 2009 J. Nucl. Mater. 390-391 1

[2] Rudakov D L et al. 2008 Rev. Sci. Instrum. 79 10F303

[3] Neu 2007 Plasma Phys.Control Fus. 49 B59 
[4] Sharpe K et al. 2003 J. Nucl. Mater. 313-316 455

[5] Mayer M et al. 2009 J. Nucl. Mater. 390-391 538

[6] Rohde V 2008 Final EFDA TW6-TPP-DUSMEAS Final Report

[7] Mayer M et al. this conference C3

[8] McCracken G M 1980 J. Nucl. Mater. 93-94 3

[9] Daalder J E 1976 J.Phys.D 92379

[10] Maier H 2004 J. Nucl. Mater. 335515

[11] Herrmann A et al. 2009 J. Nucl. Mater. 390-391 747

[12] Hong S et al submitted to Nuclear Fusion

[13] Smirnov R D et al 2009 Plasma Phys.Control Fus. 51055017 


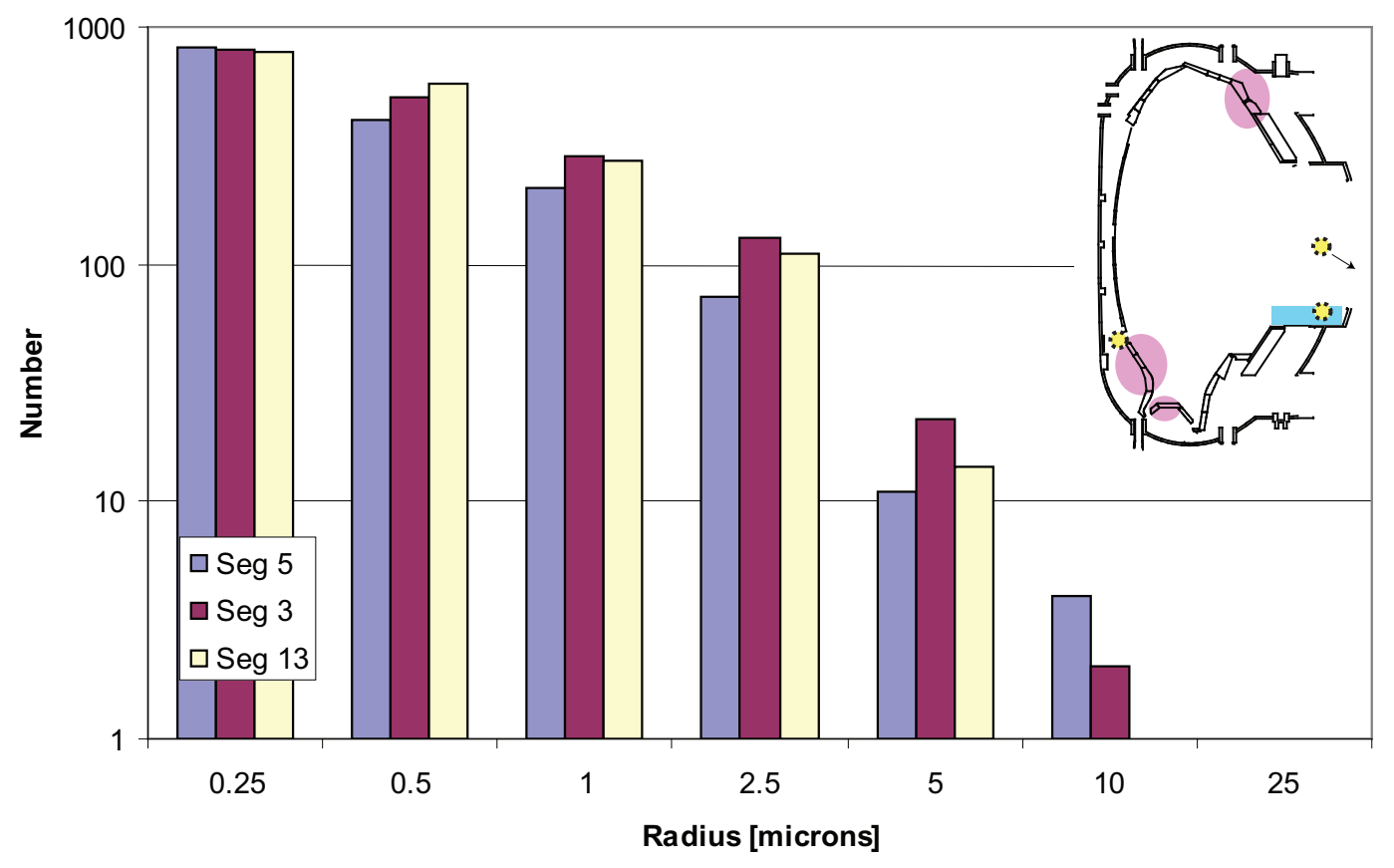

Figure 1: Distribution of the radii of the collected particles for the 3 collectors at different toroidal positions in campaign 2008. The insert shows a cross section of AUG with the polodial position of the collectors as rectangle and arc traces as ellipses.

Distribution of the radii of the collected particles for the 3 collectors at different toroidal positions in campaign 2008. The insert shows a cross section of AUG with the 3 polodial positions of the collectors as circles with dashed lines, the area used for extrapolation as rectangle and of the arc traces as filled ellipses. 


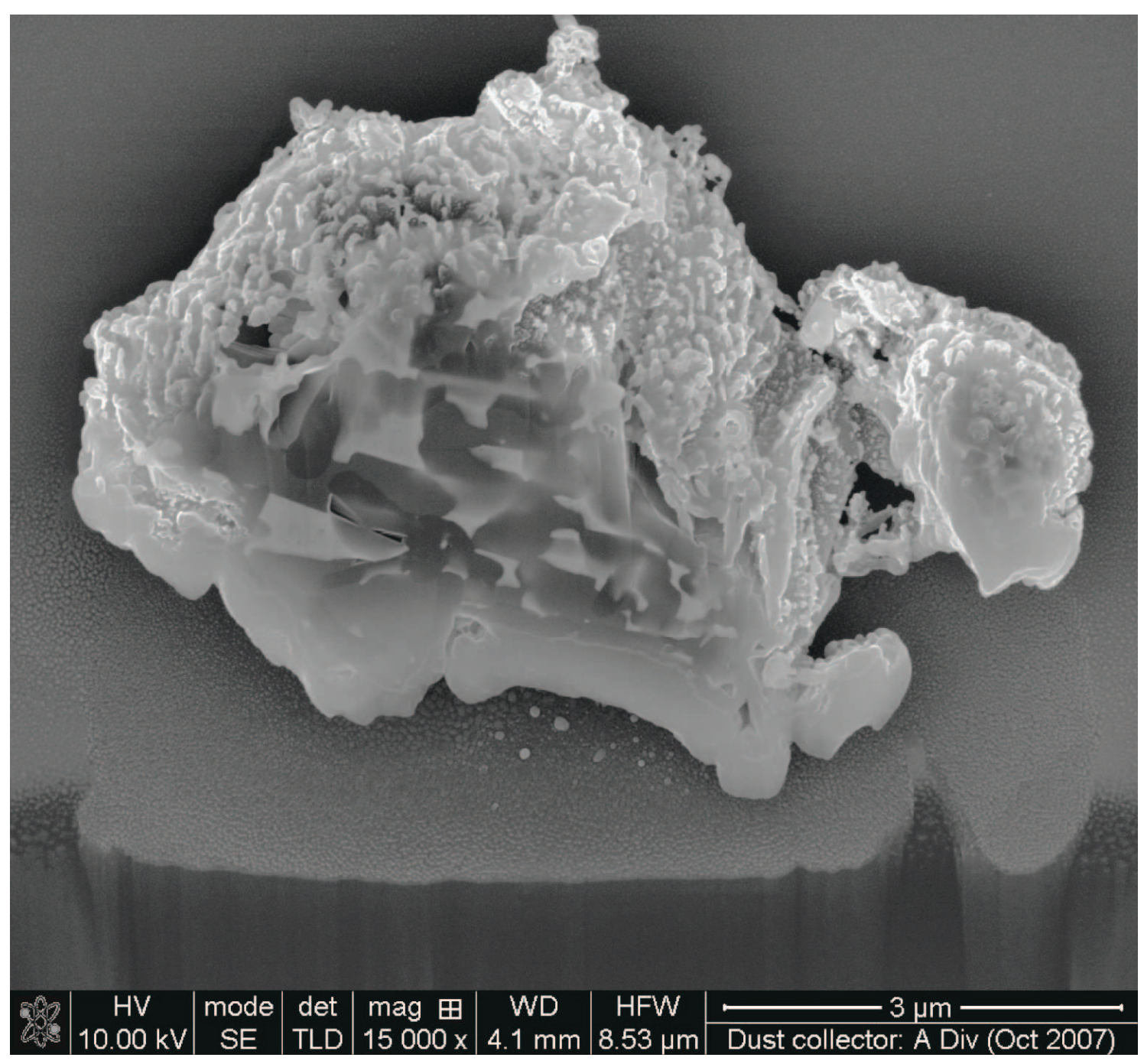

Figure 2: SEM image of an agglomerate collected in campaign 2007 at the low field side. To investigate the internal structure, the particle was partly cut using a focused ion beam. The tiny structured on the outer surface of the particle appears bright. On the cross-section, the bright spots consist of tungsten, while the dark appearing matrix consists of carbon and boron. 

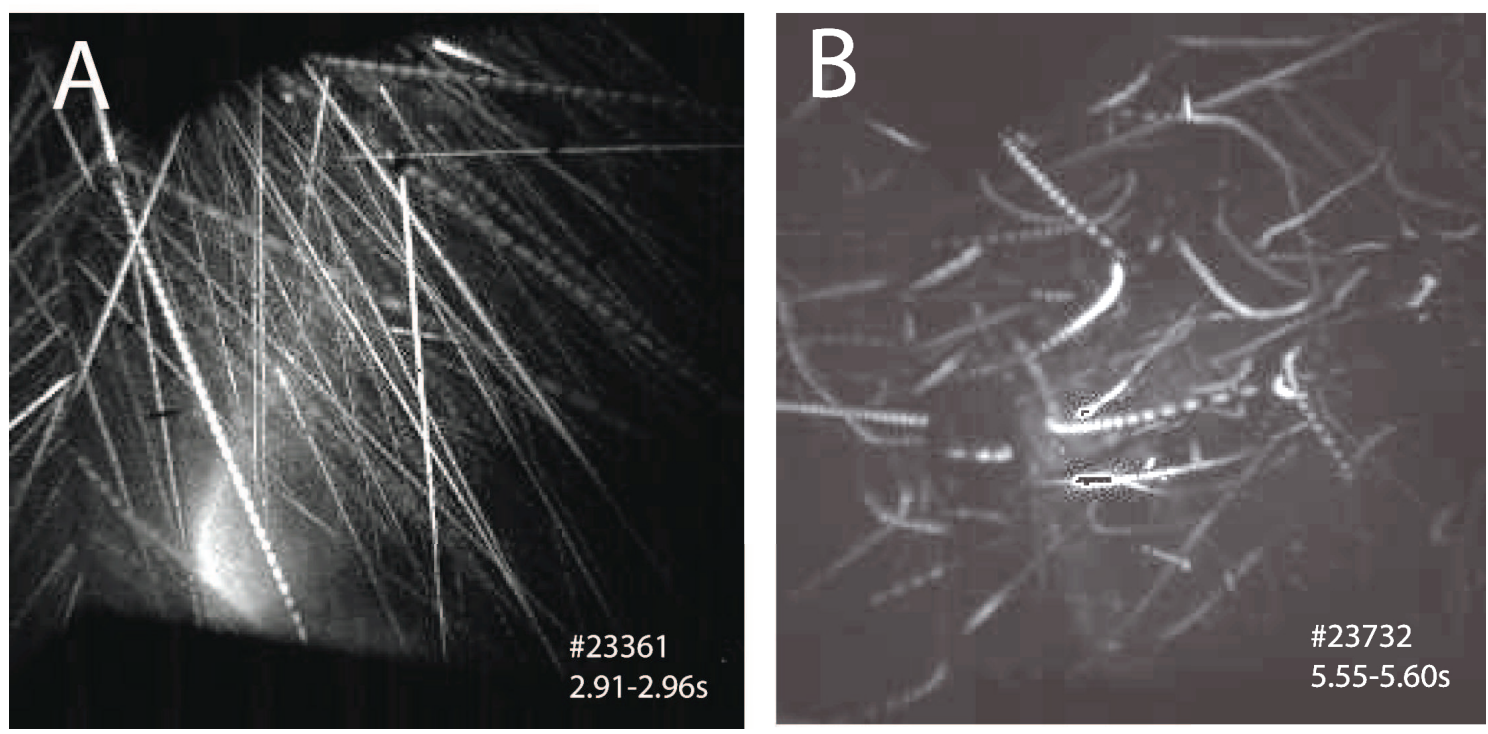

Figure 3: Trajectories of dust particles observed by the fast camera in campaign 2008. A: dust released after a disruption. B: trajectories during a high clearance discharge. The picture shows the maximal intensity of each pixel during the indicated time period of the discharge. 